\title{
UTILIZACIÓN DE LOS EQUIPOS DE PROTECCIÓN INDIVIDUAL FRENTE AL RIESGO BIOLÓGICO POR EL PERSONAL SANITARIO
}

\author{
ANGELINA CONSTANS AUBERT, \\ ROSA M ${ }^{\mathrm{a}}$ ALONSO ESPADALÉ, JOAQUÍN PÉREZ NICOLÁS \\ Centro Nacional de Condiciones de Trabajo. \\ Instituto Nacional de Seguridad e Higiene en el Trabajo. Barcelona.
}

\section{RESUMEN}

El objetivo de este trabajo ha sido obtener información sobre el nivel de utilización de los equipos de protección individual (EPI) frente al riego biológico entre el personal que desarrolla su actividad profesional en centros sanitarios. Para ello se preparó un cuestionario, que previamente validado se aplicó a un colectivo de distintos centros sanitarios de Cataluña durante los años 2003/2004. La información obtenida en este estudio revela que la utilización de los EPI frente al riesgo biológico, entre el personal que desarrolla su actividad en centros sanitarios es elevada, aunque también evidencia que todavía es necesario corregir algunos de los procedimientos de trabajo que se siguen en dichos centros y que es necesario mejorar algunos puntos de la gestión; también es una asignatura pendiente una mejor formación e información de los trabajadores para una correcta utilización de los EPI.

Finalmente, en el apartado correspondiente, se presenta una revisión exhaustiva de las Normas Técnicas relacionadas con los EPI, ropa de protección y calzado frente a microorganismos.

\section{PALABRAS CLAVES}

Riesgo biológico, protecciones personales, centros sanitarios

\begin{abstract}
The aim of this paper is to get information about the use of the PPE in front of biological risk in health care workers. A questionnaire has been prepared and, previous validation, has been applied to a set of hospitals in Catalonia area during 2003-2004. Information obtained indicates that the use of PPE in front of biological risk is high, but it is necessary to change some working procedures and to improve the management of PPE. It should also be improved information and worker's training for the correct use of PPE. Finally, a review of technical regulations related to PPE, protection clothes and shoes in front of microorganisms is presented.
\end{abstract}

\section{KEY WORDS}

Biological risk, personal protection equipment, hospitals 


\section{INTRODUCCIÓN}

La protección de los trabajadores frente a los riesgos relacionados con la exposición a agentes biológicos es una de las herramientas para garantizar la seguridad y la salud de los mismos. Por agente biológico se entiende los microorganismos, con inclusión de los genéticamente modificados, cultivos celulares y endoparásitos humanos, susceptibles de originar cualquier tipo de infección, alergia o toxicidad (Real Decreto 664/1997). La definición formal de agente biológico incluye: bacterias, hongos, parásitos y virus. Sin embargo, este concepto es actualmente más amplio, ya que dentro del grupo de los virus, se incluyen agentes no clasificados asociados a encefalopatías espongiformes transmisibles (priones o proteínas priónicas).

Algunas de las enfermedades originadas por agentes biológicos pueden producirse en el mundo laboral en general, pero es en el ambiente laboral sanitario donde estas enfermedades adquieren una especial relevancia por la mayor posibilidad de contagio, como ocurre con la hepatitis $\mathrm{B}$, la hepatitis $\mathrm{C}$, el síndrome de inmunodeficiencia adquirida (SIDA) y la tuberculosis. Sin embargo, hay que destacar que la prevalencia de estas enfermedades ha disminuido drásticamente gracias al uso de eficaces medidas de prevención.

Existen tres tipos distintos de medidas para hacer frente al riesgo biológico:

- Medidas físicas: destinadas a evitar el contacto con los agentes, sobre las bases de: confinamiento (cabinas de seguridad biológica), barreras frente al contacto dérmico o mucosas (guantes, pantallas faciales, gafas) y barreras frente a la entrada por vía respiratoria (máscaras y mascarillas con filtro o autofiltrantes). Algunos autores incluyen dentro de este último tipo de medidas la utilización de medios físicos para desinfección y esterilización, como son el calor, las radiaciones ultravioletas y las radiaciones ionizantes.

- Medidas químicas: hacen referencia a la utilización de desinfectantes y esterilizantes químicos.

- Medidas biológicas: contemplan la vacunación o inmunización activa.

La experiencia en la prevención frente al riesgo biológico indica que se suele actuar de manera com- binada, prevaleciendo en cada caso la medida o medidas más eficaces y más seguras sobre la base del principio de que el riesgo se debe eliminar siempre, y que cuando ello no sea posible, se reducirá al máximo. Al conjunto de estas medidas deben sumarse las medidas higiénicas de tipo personal sobre limpieza y mantenimiento tanto de la ropa de trabajo como de los equipos de protección, así como una serie de prohibiciones en el lugar de trabajo, como por ejemplo comer, beber, fumar, cambio de ropa y de equipos de protección individual (EPI), entre otros.

La necesidad de utilizar EPI frente a una determinada situación de riesgo biológico en un centro sanitario deriva de la imposibilidad técnica o económica de instalar una protección colectiva eficaz. Antes de dar por definitivamente implantada la utilización de un EPI frente a una determinada situación de riesgo, debe seguirse un proceso consistente en: definir la necesidad de su uso, llevar a cabo la elección del equipo adecuado, efectuar la adquisición, distribuirlo y normalizar y controlar su uso correcto.

El objetivo principal del estudio, ha sido obtener información sobre la utilización de los EPI frente al riesgo biológico entre el personal que desarrolla su actividad profesional en centros sanitarios de cara a evaluar y mejorar la aplicación del RD 664/1997, concretamente en su artículo 6 "Reducción de riegos: Adopción de medidas de protección colectiva o en su defecto, de protección individual, cuando la exposición no pueda evitarse por otros medios".

En el presente trabajo se presenta el cuestionario empleado para llevar a cabo la encuesta, se muestra un resumen de los resultados obtenidos en la aplicación de dicha encuesta en distintos centros sanitarios de Cataluña durante los años 2003/2004 y se presenta una revisión exhaustiva de las Normas Técnicas relacionadas con los EPI, ropa de protección y calzado frente a microorganismos.

\section{MATERIAL Y MÉTODOS}

El estudio se ha realizado mediante la aplicación de un cuestionario (Tabla I), previamente validado, mediante un pre-test en 8 centros sanitarios con un cuestionario previo, con la finalidad de comprobar su viabilidad e incluir las modificaciones pertinentes antes de elaborar la versión definitiva del mismo. 
Tabla I. Cuestionario utilizado en la encuesta, presentado a los distintos centros sanitarios

\begin{tabular}{|c|c|c|c|c|}
\hline & Sí & No & $\begin{array}{l}\text { No } \\
\text { sabe }\end{array}$ & $\begin{array}{c}\text { Comen- } \\
\text { tario }\end{array}$ \\
\hline $\begin{array}{l}\text { 1. Existe un compromiso por escrito por parte de la dirección del centro en cuan- } \\
\text { to a desarrollar una política eficaz de cara a la protección de la salud de los } \\
\text { trabajadores. }\end{array}$ & & & & \\
\hline $\begin{array}{l}\text { 2. Conoce el RD 664/1997 sobre la protección de los trabajadores contra } \\
\text { los riesgos relacionados con la exposición a agentes biológicos durante el } \\
\text { trabajo. }\end{array}$ & & & & \\
\hline $\begin{array}{l}\text { 3. En este centro se manipulan agentes biológicos atendiendo a lo expuesto en el } \\
\text { RD 664/1997. }\end{array}$ & & & & \\
\hline $\begin{array}{l}\text { 4. Se ha llevado a cabo la evaluación inicial de riesgos en el centro sanitario (En } \\
\text { caso de respuesta negativa pasar a la pregunta } 8 \text { ). }\end{array}$ & & & & \\
\hline 5. En la evaluación inicial de riesgos, se han incluido los riesgos biológicos. & & & & \\
\hline $\begin{array}{l}\text { 6. La evaluación de riesgos se repite periódicamente, y cada vez que se produz- } \\
\text { ca un cambio en las condiciones de trabajo que pueda afectar la salud y segu- } \\
\text { ridad de los trabajadores. }\end{array}$ & & & & \\
\hline $\begin{array}{l}\text { 7. La evaluación de riesgos se repite cuando se detecta en algún trabajador una } \\
\text { enfermedad o infección relacionada con la exposición a agentes biológicos. }\end{array}$ & & & & \\
\hline $\begin{array}{l}\text { 8. En la evaluación de riesgos se tiene en cuenta la posibilidad, de la presencia } \\
\text { de agentes potencialmente infecciosos en los pacientes, las muestras y mate- } \\
\text { riales procedentes de aquellos. }\end{array}$ & & & & \\
\hline $\begin{array}{l}\text { 9. La evaluación de riesgos ha determinado los puestos de trabajo en los que se } \\
\text { deben utilizar EPI ante la imposibilidad de eliminar o minimizar el riesgo en } \\
\text { origen. }\end{array}$ & & & & \\
\hline $\begin{array}{l}\text { 10. El centro dispone de la documentación donde se especifican las medidas de } \\
\text { prevención y de protección adoptadas como resultado de la evaluación de } \\
\text { riesgos. }\end{array}$ & & & & \\
\hline $\begin{array}{l}\text { 11. El servicio de prevención dispone de técnico/s de higiene con formación } \\
\text { específica en la prevención del riesgo biológico. }\end{array}$ & & & & \\
\hline $\begin{array}{l}\text { 12. El centro adopta las medidas higiénicas, de protección colectiva o individual } \\
\text { necesarias frente al riesgo biológico. }\end{array}$ & & & & \\
\hline $\begin{array}{l}\text { 13. Existen técnicas preventivas suficientes para evitar la exposición de los tra- } \\
\text { bajadores en el foco emisor o en el medio de propagación. }\end{array}$ & & & & \\
\hline
\end{tabular}




\begin{tabular}{|c|c|c|c|c|}
\hline & Sí & No & $\begin{array}{l}\text { No } \\
\text { sabe }\end{array}$ & $\begin{array}{c}\text { Comen- } \\
\text { tario }\end{array}$ \\
\hline $\begin{array}{l}\text { 14. En los procedimientos de trabajo esta descrita la gestión de los re } \\
\text { tarios. }\end{array}$ & & & & \\
\hline $\begin{array}{l}\text { 15. En los procedimientos de trabajo están descritos los posibles } \\
\text { exposiciones accidentales a agentes biológicos. }\end{array}$ & & & & \\
\hline $\begin{array}{l}\text { 16. Los trabajadores del centro conocen los procedimientos de trab } \\
\text { accidentes con riesgo biológico (pinchazos, cortes, salpicaduras }\end{array}$ & & & & \\
\hline $\begin{array}{l}\text { 17. Se dispone de EPI u otras medidas de protección frente a estas } \\
\text { accidentales. }\end{array}$ & & & & \\
\hline 18. Las zonas donde existe riesgo biológico están señalizadas. & & & & \\
\hline 19. Se ha formado a los trabajadores sobre la utilización de EPI. & & & & \\
\hline $\begin{array}{l}\text { 20. Se realizan inspecciones de seguridad para verificar que la gesti } \\
\text { vención frente al riesgo biológico es adecuada. }\end{array}$ & & & & \\
\hline $\begin{array}{l}\text { 21. Existe un procedimiento de trabajo sobre utilización y mantenin } \\
\text { EPI. }\end{array}$ & & & & \\
\hline $\begin{array}{l}\text { 22. El usuario de los EPI no desechables es responsable de su mant } \\
\text { conservación. }\end{array}$ & & & & \\
\hline $\begin{array}{l}\text { 23. Cuando los EPI tienen más de un usuario, el servicio de prever } \\
\text { cabo su gestión. }\end{array}$ & & & & \\
\hline $\begin{array}{l}\text { 24. En la selección de los EPI se ha tenido en cuenta las disponibili } \\
\text { mercado ofrece y que cumplan la legislación correspondiente as } \\
\text { lización. }\end{array}$ & & & & \\
\hline 25. Los representantes de los trabajadores participan en la selección & & & & \\
\hline $\begin{array}{l}\text { 26. En la elección de los EPI se han tenido en cuenta las caracterí } \\
\text { trabajadores. }\end{array}$ & & & & \\
\hline 27. Se suministran gratuitamente los EPI. & & & & \\
\hline 28. Se reponen los EPI cuando es necesario. & & & & \\
\hline 29. Existe una relación de los EPI asociados a cada puesto de traba & & & & \\
\hline 30. Existe una relación de los EPI suministrados a cada trabajador. & & & & \\
\hline
\end{tabular}




\begin{tabular}{|c|c|c|c|c|}
\hline & Sí & No & $\begin{array}{l}\text { No } \\
\text { sabe }\end{array}$ & $\begin{array}{c}\text { Comen- } \\
\text { tario }\end{array}$ \\
\hline 31. Existe algún documento que acredite la entrega de los EPI a los trabajadores. & & & & \\
\hline $\begin{array}{l}\text { 32. El centro sanitario dispone de fichas de seguridad biológica u otros procedi- } \\
\text { mientos de información específicos sobre la manipulación de sustancias } \\
\text { infecciosas. }\end{array}$ & & & & \\
\hline 33. Todos los trabajadores tienen acceso a los procedimientos anteriores. & & & & \\
\hline $\begin{array}{l}\text { 34. En función del área de atención sanitaria está determinado que tipo de ropa } \\
\text { de trabajo debe utilizar el personal. }\end{array}$ & & & & \\
\hline $\begin{array}{l}\text { 35. Los trabajadores disponen lugares específicos para guardar los EPI y la ropa } \\
\text { de trabajo separados de la ropa personal. }\end{array}$ & & & & \\
\hline $\begin{array}{l}\text { 36. Existe la prohibición de que los trabajadores se lleven la ropa de trabajo a su } \\
\text { domicilio. }\end{array}$ & & & & \\
\hline $\begin{array}{l}\text { 37. Existe la prohibición de que los trabajadores se lleven los EPI a su domici- } \\
\text { lio. }\end{array}$ & & & & \\
\hline $\begin{array}{l}\text { 38. El centro se encarga del lavado, descontaminación o si es necesario de la des- } \\
\text { trucción de la ropa de trabajo. }\end{array}$ & & & & \\
\hline $\begin{array}{l}\text { 39. El centro se encarga del lavado, descontaminación o si es necesario de la des- } \\
\text { trucción de los EPI. }\end{array}$ & & & & \\
\hline $\begin{array}{l}\text { 40. En el caso de que el centro tenga contratado el servicio del lavado, descon- } \\
\text { taminación o destrucción de la ropa de trabajo y de los EPI, se asegura que } \\
\text { el envío se haga correctamente en recipientes cerrados y etiquetados con las } \\
\text { advertencias necesarias. }\end{array}$ & & & & \\
\hline $\begin{array}{l}\text { 41. El centro dispone de instrucciones específicas sobre la forma de desconta- } \\
\text { minar y desinfectar. }\end{array}$ & & & & \\
\hline $\begin{array}{l}\text { 42. Se utilizan equipos de protección respiratoria que cumplan con el RD } \\
1407 / 1992 \text { sobre la comercialización de EPI cuando la protección requerida } \\
\text { es contra la inhalación de microorganismos. }\end{array}$ & & & & \\
\hline $\begin{array}{l}\text { 43. El centro dispone de guantes desechables, tallas adecuadas, adaptables y a } \\
\text { disposición de los trabajadores siempre que sean necesarios. }\end{array}$ & & & & \\
\hline $\begin{array}{l}\text { 44. El centro dispone de guantes sintéticos a disposición de los trabajadores con } \\
\text { alergia a los guantes de látex. }\end{array}$ & & & & \\
\hline $\begin{array}{l}\text { 45. Se utilizan guantes cuando se manipula sangre y derivados, líquidos corpo- } \\
\text { rales, excreciones, secreciones y pacientes con la piel dañada, etc. }\end{array}$ & & & & \\
\hline $\begin{array}{l}\text { 46. Se utilizan guantes cuando se realizan procedimientos de accesos a vasos } \\
\text { sanguíneos. }\end{array}$ & & & & \\
\hline $\begin{array}{l}\text { 47. Está establecido como y cuando deben cambiarse los guantes, así como el } \\
\text { lavado de las manos. }\end{array}$ & & & & \\
\hline
\end{tabular}




\begin{tabular}{|c|c|c|c|c|}
\hline & Sí & No & $\begin{array}{l}\text { No } \\
\text { sabe }\end{array}$ & $\begin{array}{c}\text { Comen- } \\
\text { tario }\end{array}$ \\
\hline 48. Está establecido cuando debe utilizarse protección ocular. & & & & \\
\hline $\begin{array}{l}\text { 49. Se aplica el protocolo de vigilancia sanitaria específica para los trabajadores } \\
\text { expuestos a agentes biológicos. }\end{array}$ & & & & \\
\hline 50. Se informa al personal de las ventajas e inconvenientes de la vacunación. & & & & \\
\hline $\begin{array}{l}\text { 51. El centro proporciona de forma gratuita vacunas eficaces al personal expues- } \\
\text { to a agentes biológicos. }\end{array}$ & & & & \\
\hline $\begin{array}{l}\text { 52. Se ha notificado a la autoridad laboral la utilización de agentes biológicos de } \\
\text { los grupos } 2,3 \text { y } 4 \text {. }\end{array}$ & & & & \\
\hline $\begin{array}{l}\text { 53. Se adoptan medidas preventivas adicionales en cuanto a la utilización de EPI } \\
\text { en servicios con pacientes que se sospeche que están contaminados con } \\
\text { agentes biológicos de los grupos } 3 \text { y } 4 \text {. }\end{array}$ & & & & \\
\hline $\begin{array}{l}\text { 54. Se proporciona a los trabajadores y a sus representantes la formación e infor- } \\
\text { mación sobre las medidas a adoptar relacionadas con la exposición a agen- } \\
\text { tes biológicos. }\end{array}$ & & & & \\
\hline $\begin{array}{l}\text { 55. En caso de que se introduzcan cambios en los procedimientos de trabajo que } \\
\text { impliquen modificaciones respecto a la utilización y mantenimiento de los } \\
\text { EPI, se informa al personal. }\end{array}$ & & & & \\
\hline
\end{tabular}


El cuestionario contiene dos bloques diferenciados:

A. Datos de identificación del centro: De respuesta opcional.

B. Cuestionario: Destinado a recabar información sobre el grado de utilización de los EPI mediante 55 preguntas de tipo test con respuesta excluyente y un apartado de comentarios para cada una de ellas.

Las preguntas estaban dirigidas a obtener información, principalmente, sobre los siguientes temas:

1. Política preventiva (preguntas 1-13, 18, 20, 49-53)

2. Gestión de los EPI (preguntas 14, 21-30, 33, $37,39-41)$

3. Guantes (preguntas 43-47)

4. Protección respiratoria (pregunta 42)

5. Protección ocular (pregunta 48)

6. Ropa de protección / de trabajo (preguntas 34$36,38,40)$

7. Equipos para situaciones de emergencia (preguntas 15-17)

8. Formación (preguntas 19, 54)

9. Información (preguntas 50, 55)

10. Documentación (preguntas 31-32)

El estudio de campo se llevó a cabo en el periodo de tiempo transcurrido entre septiembre de 2003 a abril de 2004. Para ello se remitieron 198 cuestionarios por correo ordinario y/o electrónico dirigidos a los Servicios de Prevención de centros sanitarios públicos, privados y concertados. El área geográfica en la que se realizó el estudio fue Cataluña.

\section{RESULTADOS}

De los 198 cuestionarios enviados a los diferentes centros sanitarios, se recibió respuesta por parte de 45 , lo que significa un nivel de respuesta del $22,73 \%$.

El análisis de la información obtenida sobre los centros que contestaron el primer bloque muestra los resultados que se resumen a continuación.

\section{- Tipo de centro sanitario}

Un $85,7 \%$ de los centros son de tipo concertado, la mayoría pertenecientes a la provincia de Tarragona. No se recibió respuesta por parte de ningún centro perteneciente a la provincia de Lérida.

\section{- Tamaño del centro sanitario}

Clasificando el tamaño del centro según el número de camas, el mayor nivel de respuestas se obtuvo por parte de los centros sanitarios cuyo tamaño pertenece al grupo de entre 100 y 200 camas $(33,3 \%)$, seguido del grupo de entre 201 y 300 camas $(23,3 \%)$.

\section{- Ámbito de aplicación sanitaria}

Otro aspecto a comentar es el área de atención sanitaria en la que se ha aplicado el cuestionario; el $87,5 \%$ de las respuestas indica que se aplicó en todas las áreas del centro. Posiblemente en este hecho influyó que la mayoría de los cuestionarios fueron cumplimentados directamente por el Servicio de Prevención.

\section{- Plantilla}

La plantilla se ha clasificado, en función del número de trabajadores, en tres grupos:

Grupo 1: 50-249 trabajadores

Grupo 2: 250-500 trabajadores

Grupo 3: $>500$ trabajadores

La mayoría de los centros que respondieron $(80 \%)$ corresponden a centros de hasta 500 trabajadores. (Figura 1).

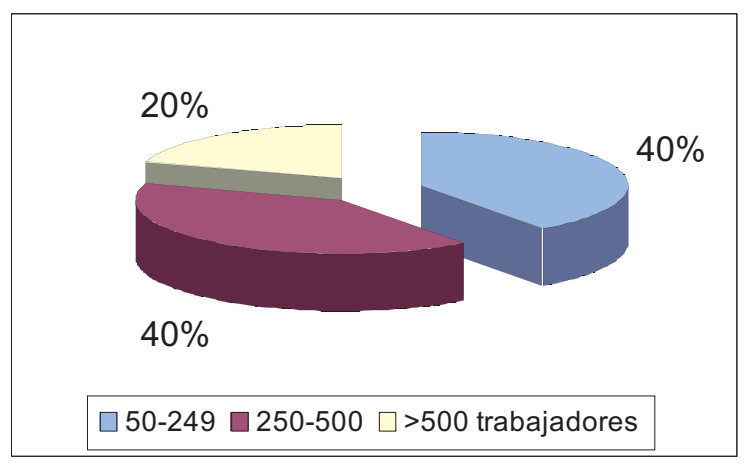

Figura 1. Tamaño del centro sanitario según la plantilla 


\section{CUESTIONARIO}

El análisis de la información obtenida sobre los centros que contestaron el segundo bloque, se realizó destacando los aspectos que pueden tener mayor interés preventivo. Los resultados de este bloque, 55 preguntas en total, se comentan a continuación.

\section{- Política preventiva}

El $86,7 \%$ de los centros sanitarios desarrollan una política preventiva correcta referente al cumplimiento del RD 664/1997. En este sentido, es interesante destacar que en el $100 \%$ de los centros, la evaluación de riesgos ha incluido los riesgos biológicos y ha determinado los puestos de trabajo en los que es necesaria la utilización de EPI frente a este riesgo ante la imposibilidad de establecer medidas de protección colectiva

No obstante, únicamente el $50 \%$ de los centros realiza inspecciones de seguridad para verificar que la gestión frente al riesgo biológico es adecuada y sólo en el 56,3\% de los centros están correctamente señalizadas las zonas en donde existe riesgo biológico.

\section{- Gestión de los equipos de protección individual}

El 74,2\% de los centros responde afirmativamente a las preguntas sobre los diferentes mecanismos de gestión que llevan a cabo; sin embargo, se observan deficiencias al plantear que el EPI pueda tener más de un usuario, en cuyo caso no queda claro quién se responsabiliza de su gestión. (Figura 2).

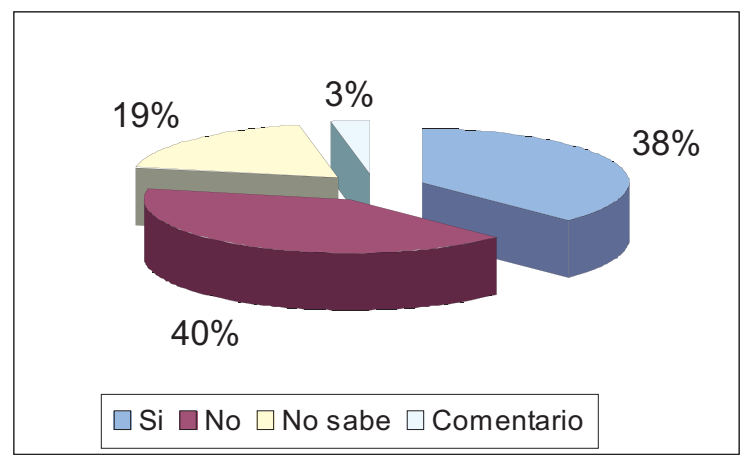

Figura 2. Gestión de los equipos de protección individual con más de un usuario

Además hay que destacar que en el 50\% de los centros afirma que los representantes de los trabajadores participan en la elección de los EPI frente al
$44 \%$ que no participan y el $6 \%$ no sabe quien se encarga de la elección. Respecto a la existencia de un procedimiento de trabajo para la utilización y el mantenimiento de los mismos contestan afirmativamente un 53,2\% de los centros (Figura 3). Por otro lado, en el $50 \%$ de los centros existe una relación de los EPI asociados a cada puesto de trabajo y únicamente el 28,1\% tiene una relación de los EPI suministrados a cada trabajador.

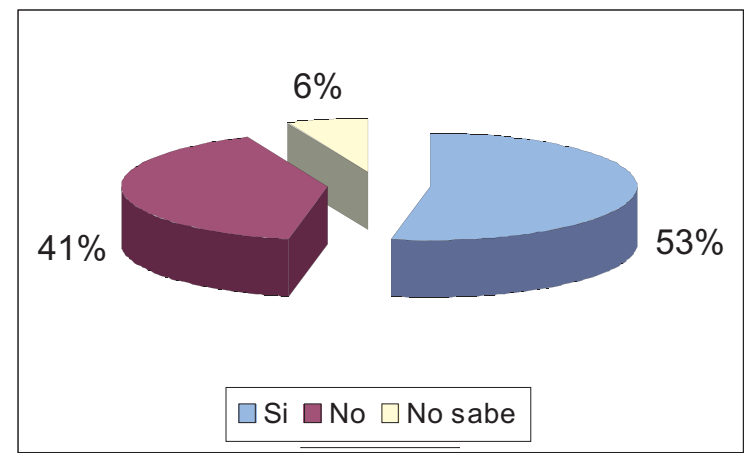

Figura 3. Existencia de un procedimiento de trabajo para el uso y el mantenimiento de los equipos de protección individual

\section{- Guantes}

La mayoría de los centros, el 96,9\%, dispone de guantes desechables, de diversas tallas y materiales, adecuados a los diferentes procedimientos de trabajo (manipulación de sangre, líquidos corporales, etc.). Un $93,8 \%$ de los centros responde afirmativamente a la pregunta de si está establecido como y cuando deben cambiarse los guantes, así como el lavado de las manos.

Otro aspecto a comentar, dada la problemática derivada de su utilización, son los guantes de látex. Un 3,1\% de los centros responde que desconocen si el centro dispone de guantes alternativos al látex.

\section{- Protección respiratoria}

El 93,8\% de los centros utilizan equipos de protección respiratoria válidos contra la inhalación de microorganismos y que cumplen con la normativa de comercialización de los EPI.

\section{- Protección ocular}

En el 78\% de los centros esta establecido cuando debe utilizarse protección ocular mientras que en el $16 \%$ no está establecido y en el $6 \%$ se desconoce. 


\section{- Ropa de protección/de trabajo}

En el 93,8\% de los centros, está determinado el tipo de ropa de trabajo que debe utilizarse en función del área de trabajo. Sin embargo, aunque la mayoría de los centros $(96,9 \%)$ se encargan del lavado de la ropa, en un $15,6 \%$ no existe la prohibición expresa de que el trabajador pueda llevarse la ropa de trabajo a su domicilio. (Figura 4).

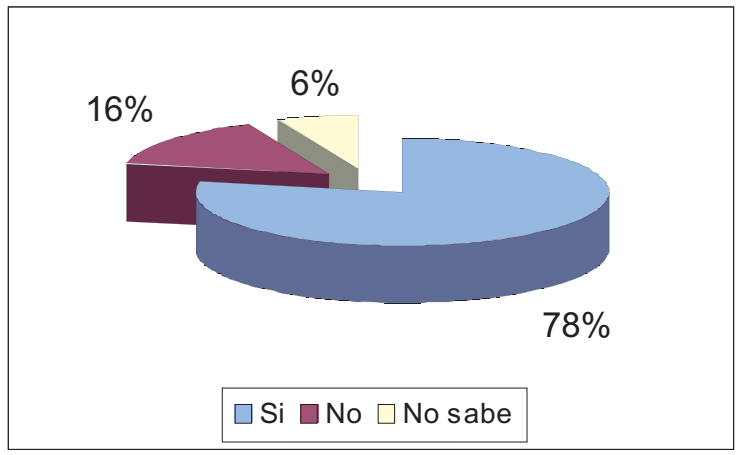

Figura 4. Prohibición de llevarse la ropa de trabajo a su domicilio

En caso de que el lavado y/o posterior tratamiento de la ropa se realice por un servicio externo, un $65,6 \%$ de los centros afirma disponer de mecanismos para asegurarse de que el envío se hace en recipientes cerrados y etiquetados.

\section{- Equipos para situaciones de emergencia}

En el $84,4 \%$ de los centros afirma que en sus procedimientos de trabajo están descritos los posibles riesgos de exposiciones accidentales a riesgos biológicos. El 96,9\% de los centros responde que el personal conoce los procedimientos de trabajo frente a accidentes con riesgo biológico (pinchazos, cortes, salpicaduras, etc.). E1 90,6\% de los centros dispone de EPI u otras medidas de prevención para situaciones de emergencia.

\section{- Formación}

En el 90,6\% de los centros se imparte formación a los trabajadores y a sus representantes sobre la utilización de los EPI y el 84,4\% proporciona también la formación y la información de las medidas a adoptar relacionadas con la exposición a agentes biológicos.

\section{- Información}

Se ha estudiado el sistema de información en base a dos preguntas: la primera, respecto a la vacu- nación, el 93,8\% de los centros informa a los trabajadores de las ventajas e inconvenientes de la vacunación; la segunda, respecto a si se informa al personal cuando se introducen cambios que implican modificaciones sobre la utilización de los EPI, el $90,6 \%$ de los centros responde afirmativamente.

\section{- Documentación}

En el 40,6\% de los centros existe algún documento que acredite la entrega de los EPI a los trabajadores, mientras que en el $53,1 \%$ no dispone de dicha documentación. El 78,1\% dispone de fichas de seguridad biológica u otros procedimientos de información específicos sobre la manipulación de sustancias infecciosas. (Figura 5).

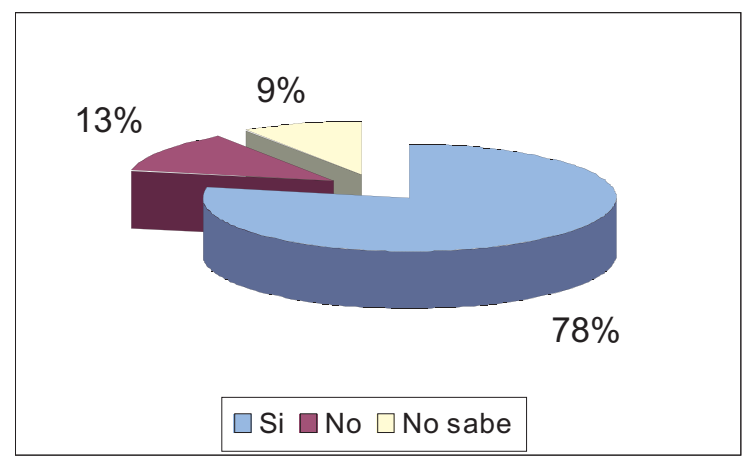

Figura 5. Existencia de ficha de seguridad biológica u otro procedimiento de información

\section{DISCUSIÓN}

La información obtenida en este estudio sobre la utilización de los EPI frente al riesgo biológico entre el personal que desarrolla su actividad en centros sanitarios demuestra que el cumplimiento del artículo 6 del RD 664/1997 es elevado, aunque es evidente que es necesario corregir en algunos puntos que se comentan a continuación.

Es importante resaltar que un elevado número de centros sanitarios observan una política preventiva correcta, aunque con algunas deficiencias en cuanto a la señalización y a las inspecciones de seguridad para verificar la gestión frente al riesgo biológico.

En cuanto a la gestión, aproximadamente un $75 \%$ de los centros responde afirmativamente a la aplicación de algún mecanismo de gestión, observándose algunas deficiencias sobre todo cuando el EPI pueda tener más de un usuario, ya que en muchos casos no queda claro quien se responsabiliza de su gestión. En relación con el hecho de que los trabajadores o sus 
representantes participen en la elección de los EPI y que exista un procedimiento de trabajo sobre su utilización, solamente un 50\% participa en la elección y un $53 \%$ de los centros disponen de un procedimiento de trabajo.

El estudio demuestra que la mayoría de los centros disponen y tienen establecido cuando deben utilizarse los EPI específicos tales como: guantes, protecciones respiratorias, protecciones oculares, ropa de protección o de trabajo así como equipos para situaciones de emergencia.

En más de un $90 \%$ de los centros se imparte formación y se informa a los trabajadores tanto cuanto se introducen cambios en la utilización de los EPI como también sobre las ventajas e inconvenientes de la vacunación.

Sin embargo en cuanto a la documentación, el porcentaje baja hasta el $40 \%$ la existencia de algún documento que acredite la entrega de los EPI a los trabajadores y a un $78 \%$ sobre procedimientos de información específicos sobre manipulación de sustancias infecciosas.

Los resultados obtenidos en este estudio, así como la reciente aparición de normativa técnica específica sobre EPI, ropa de protección y calzado de protección contra microorganismos inciden en la necesidad de seguir trabajando sobre este tema.

Finalmente cabe resaltar la importancia de la labor de formación e información, ya que el personal expuesto a riesgos biológicos debe diferenciar claramente los EPI, de la ropa de trabajo y de los equipos destinados a la protección del producto (paciente o muestra manipulada), usándose éstos últimos como protecciones personales frente al riesgo biológico, cuando en la mayoría de situaciones no sólo no son eficaces sino que producen una situación de falsa seguridad.

\section{NORMATIVA TÉCNICA}

Se incluye a continuación una relación actualizada de la Normativa Técnica, directamente relacionada con los EPI, ropa de protección y calzado contra microorganismos y también las normas relacionadas con los guantes de uso médico.

UNE-EN 132:1999. Equipos de protección respiratoria. Definiciones de términos y pictogramas.

UNE-EN 133:2002. Equipos de protección respiratoria. Clasificación.
UNE-EN 134:1998. Equipos de protección respiratoria. Nomenclatura de los componentes.

UNE-EN 143:2001. Equipos de protección respiratoria. Filtros contra partículas. Requisitos, ensayo, marcado.

UNE-EN 149:2001. Equipos de protección respiratoria. Medias máscaras filtrantes de protección contra partículas. Requisitos, ensayo, marcado.

UNE-EN 529:2006. Equipos de protección respiratoria. Recomendaciones sobre selección, uso, cuidado y mantenimiento.

UNE-EN 374-1:2004. Guantes de protección contra los productos químicos y los microorganismos.

\section{Parte 1: Terminología y requisitos de las presta- ciones.}

UNE-EN 374-2:2004. Guantes de protección contra los productos químicos y los microorganismos.

\section{Parte 2: Determinación de la resistencia a la penetración.}

UNE-EN 374-3:2004/AC:2006. Guantes de protección contra los productos químicos y los microorganismos.

Parte 3: Determinación de la resistencia a la permeación por productos químicos.

UNE-EN 420:1995. Requisitos generales para los guantes.

UNE-EN-455-1:2001. Guantes médicos para un solo uso. Parte 1: Requisitos y ensayos para determinar la ausencia de agujeros.

UNE-EN-455-2:2001. Guantes médicos para un solo uso. Parte 2: Requisitos y ensayos para determinar las propiedades físicas.

UNE-EN-455-3:2000. Guantes médicos para un solo uso. Parte 3: Requisitos y ensayos para la evaluación biológica.

UNE-EN 340:2004. Ropas de protección. Requisitos generales. 
UNE-EN 14126: 2004/AC:2006. Ropa de protección: requisitos y métodos de ensayo para la ropa de protección contra agentes biológicos.

UNE-EN ISO 22612:2005. Ropa de protección contra agentes infecciosos. Método de ensayo de la resistencia a la penetración microbiana en seco.

PNE prEN 13832-1. Calzado de protección contra agentes químicos y microorganismos. Parte 1: Terminología y métodos de ensayo.
PNE prEN 13832-2. Calzado de protección contra agentes químicos y microorganismos. Parte 2: Calzado de protección contra agentes químicos rociados.

PNE prEN 13832-3. Calzado de protección contra agentes químicos y microorganismos. Parte 3: Calzado de alta protección contra agentes químicos. 